Journal: American Journal of Audiology

\title{
Knowledge, beliefs and practices of Australian audiologists in addressing the mental health needs of adults with hearing loss
}

\author{
Rebecca J Bennett*, ${ }^{\dagger}$, \\ Carly J Meyer ${ }^{\circ}$, \\ Brooke Ryan ${ }^{\text {, }}$ \\ Caitlin Barr^, \\ Emma Laird^, \\ Robert H Eikelboom ${ }^{*+,},+$
}

* Ear Science Institute Australia, Subiaco, Australia, ${ }^{\dagger}$ Ear Sciences Centre, The University of Western Australia, Nedlands, Australia, ${ }^{\circ}$ School of Health and Rehabilitation Sciences, University of Queensland, Brisbane, Australia, ${ }^{\ddagger}$ Department of Speech Language Pathology and Audiology, University of Pretoria, Pretoria, South Africa, ^ Department of Audiology and Speech Pathology, University of Melbourne, Melbourne, Australia

Corresponding author: Dr Rebecca J Bennett, Ear Science Institute Australia, Suite 1, Level 2, 1 Salvado Road, Subiaco, WA 6008, Australia. Tel: +61 863804900 . Fax: +61 863804901. Email: bec.bennett@earscience.org.au

Conflict of Interest: The authors declare that they have no competing interests.

Funding: This work was funded by the Ear Science Institute Australia. 


\begin{abstract}
Purpose: Emotional and mental health is essential to overall health but there has been little research on how to approach emotional and mental health in the audiology setting. This study provides a preliminary investigation into the current knowledge, beliefs and practices of Australian audiologists in addressing the emotional and mental health needs of adults with hearing loss.
\end{abstract}

Method: A 22-item survey using open and closed-ended questions was completed by 95 Australian audiologists using a cross-sectional study design.

Results: Two thirds of audiologists described being under-confident and lacking the skills required to provide emotional support to people with hearing loss. Barriers to delivering emotional support included feeling out of their depth (56.6\%), time/caseload pressures (55.3\%), and the perception that the provision of emotional support was not within an audiologist's scope of practice (31.6\%). Audiologists described a desire to refer clients to mental health professionals, yet highlighted significant barriers, including not knowing who to refer to $(54.7 \%)$, when to make a referral $(49.3 \%)$, or how to make a referral $(38.6 \%)$. Audiologists overwhelmingly (96\%) indicated that they would like to develop their knowledge and skills associated with the provision of emotional and mental health support in the audiological setting.

Conclusion: Knowledge, skills and time were identified as the key areas that require attention in order to allow audiologists to address the emotional and mental health needs of adults with hearing loss. 


\section{INTRODUCTION}

Mental health disorders such as depression and anxiety are major public health issues due to their high prevalence and poor outcomes (Byers, Yaffe, Covinsky, Friedman, \& Bruce, 2010). Approximately $45 \%$ of adults experience mental health concerns during their life, with late-life depression (the onset of depression in the later years of life) affecting between 10 to $15 \%$ of Australians over the age of 65 years (Australian Bureau of Statistics, 2008). The number of older adults (over 55 years of age) experiencing psychiatric disorders in the USA is increasing; $11.4 \%$ have an anxiety disorder, $6.8 \%$ a mood disorder, $5.6 \%$ major depression, and $14.3 \%$ have one or more personality disorders (Reynolds, Pietrzak, El-Gabalawy, Mackenzie, \& Sareen, 2015). Hearing loss and the associated breakdown of communication affects older adults' ability to socially engage (Broese van Groenou, Hoogendijk, \& van Tilburg, 2013; Kramer, Kapteyn, Kuik, \& Deeg, 2002; Mikkola et al., 2016), subsequently contributing to social and emotional isolation (Mick, Kawachi, \& Lin, 2014; Weinstein, Sirow, \& Moser, 2016). Social isolation associated with hearing is considered to be a major contributor to late-life mental health symptoms (Pronk et al., 2013; Rutherford, Brewster, Golub, Kim, \& Roose, 2017), with hearing loss increasing the risk of depression by $47 \%$ in older adults (Lawrence et al., 2019).

Audiologists providing services to adults with hearing loss are frequently put into the role of counsellor as clients frequently raise emotional concerns associated with hearing loss and audiological rehabilitation (English, Naeve-Velguth, Rall, Uyehara-Isono, \& Pittman, 2007; Meibos, 2019; Meibos, Munoz, Schultz, Price, Whicker, Caballero, \& Graham, 2017). Unlike practising psychologists, audiologists are not formally trained or qualified to provide 
intensive therapeutic interventions for the purpose of treating individuals with mental health disorders. However, audiologists are trained to provide 'informational counselling' and 'personal adjustment counselling' (also described as emotional support or emotionfocussed counselling) (English et al., 2007; English, Rojeski, \& Branham, 2000). Where the former relates to the acquisition of knowledge and skills required for effective communication and use of assistive hearing devices, the latter describes responding to the affective component in clients' dialogue. During audiology consultations, audiologists have the opportunity to engage in dialogue with older adults regarding how their hearing loss impacts on their quality of life (e.g., personal relationships, social interactions) (Ekberg, Grenness, \& Hickson, 2014; Ekberg, Barr, \& Hickson, 2017; Grenness, Hickson, LaplanteLevesque, Meyer, \& Davidson, 2015). Through these conversations, audiologists have the opportunity to recognise and detect signs of low emotional well-being and mental health symptoms. There is therefore a potential for audiologists to play an important role in the early-detection of mental health concerns in older adults with hearing loss, to provide resources for support services, and to work with medical and mental health practitioners (i.e., psychologists and psychiatrists) to improve early intervention. However, it is not yet known if audiologists have the knowledge or skills to identify and address the emotional and mental health challenges experienced by adults with hearing loss.

Previous studies have highlighted deficiencies in allied health professionals' knowledge and skills associated with mental health symptom management. Sekhon, Douglas, and Rose (2015) investigated the beliefs, attitudes and practices of Australian speech-language pathologists in addressing psychological well-being in people with aphasia after stroke. They found that self-perception of being under-skilled was the main barrier identified to addressing mental health symptoms in people with aphasia, followed by inadequate time, 
inadequate staffing, and people with aphasia declining referral to counselling. Research points toward targeted training to improve clinicians' skills and confidence in recognising and addressing clients' mental health needs. Prior training was found to be associated with significantly higher levels of knowledge and confidence in managing psychological wellbeing in nursing staff working in residential and community aged care settings (Davison, McCabe, Mellor, Karantzas, \& George, 2009), and speech-and-language therapists (Northcott, Simpson, Moss, Ahmed, \& Hilari, 2017; Sekhon et al., 2015). The effects of mental health training on the clinical behaviours of audiologists is unknown.

This is the first study to investigate audiologists' knowledge, beliefs and practices in addressing the mental health needs of adults with hearing loss. The aims of this study were: 1) to assess audiologists' knowledge of depression, and 2) to explore beliefs and clinical practices associated with addressing the emotional and mental health needs of adults with hearing loss during audiology consultations. Given that previous studies have identified the impact of prior training and years of experience on knowledge of depression and clinical practices, this study also investigated the possible impact of personal and professional factors (age, gender, years of experience, area of speciality, location of employment, formalised training, and experiencing or knowing someone who has experienced mental health issues) on the aforementioned aims.

\section{METHODS}

A cross-sectional study was conducted to evaluate the knowledge, beliefs and practices of Australian audiologists in addressing the mental health needs of adults with hearing loss using a purposefully developed survey. Ethical approval for this study was 
granted by the Human Research Ethics Office of The University of Western Australia, and The University of Queensland's Human Research Ethics Committee.

\section{Materials}

The survey used in this study was developed based on previously published surveys used to evaluate knowledge, beliefs and practices of nurses and speech-language pathologists in addressing clients' mental health needs during routine clinical care (Davison et al., 2009; Northcott et al., 2017; Sekhon et al., 2015). The survey comprised three sections: (1) demographic and professional questions; (2) three case vignettes to explore symptom-management activities (to be reported separately); and (3) questions investigating knowledge, beliefs and practices of audiologists in addressing the emotional and mental health needs of adults with hearing loss.

Section One. Questions relating to participant demographics and clinical experience included: age, gender, years of clinical experience, location of employment, area of clinical speciality, and extent of formal training received in delivering emotional support. Participants were also asked to indicate whether they or anyone close to them had experience with mental health issues. This question was optional and participants were advised to leave it blank if they did not want to respond. Participants were also questioned whether they routinely asked their audiology clients if they were concerned about their mental well-being or have recently experienced anxiety, depression or suicidal thoughts.

Section Two. Three case vignettes were used to investigate how audiologists currently respond to symptoms of mental illness in the audiology setting (to be reported in a subsequent paper). 
Section Three. Section three included questions related to participants' knowledge of depression, as well as their beliefs and clinical behaviours regarding the provision of emotional and mental health support in the audiology setting. A group of questions evaluated knowledge of depression using 16 facts, myths and symptoms relating to depression. This section included all ten items of The Knowledge of Late-Life Depression Scale - Revised (Karantzas, Davison, McCabe, Mellor, \& Beaton, 2012) and an additional six questions developed by the authors of this study, to ensure symptoms of depression commonly associated with hearing loss were included. All 16 questions were evaluated using a four point Likert scale from " 1 = strongly disagree" to " $4=$ strongly agree" (Karantzas et al., 2012). A knowledge of depression score was calculated by adding the scores of all 16 questions, with a higher score (maximum 64) representing a higher level of knowledge. The mean scores of normative data of nurses working in aged care range from 2.46 (SD 0.83) to 3.25 (SD 0.23), with this being described as moderate levels of knowledge of late-life depression (Karantzas et al., 2012).

Participants were asked to indicate the main barriers for them in delivering emotional support to people with hearing loss. Ten possible responses were provided based on those used by Northcott et al. (2017) and Sekhon et al (2015); participants were asked to select all options that applied to them. An "other" option was also included to allow participants to provide comment on other barriers. Participants were asked whether they would be interested in developing their knowledge and skills associated with mental health and emotional well-being (Yes/No). Participants were asked to indicate what would help them to improve their delivery of emotional support to people with hearing loss. Nine options were provided based on those used by Northcott et al. (2017) and Sekhon et al 
(2015); participants were asked to select all that applied to them. An "other" option was also included to allow participants to provide comment on other barriers.

Two questions, adopted from Northcott et al. (2017), investigated experiences with working with mental health professionals. The first of these asked participants to indicate how likely they would be to refer a client with hearing loss to a mental health professional if they presented with: a) loneliness, b) anxiety, c) depressive symptoms (e.g., sadness, hopelessness, worthlessness), or d) suicidal thoughts. The second of these asked participants to indicate the main barriers to making a referral to a mental health practitioner. Nine options were provided based on those used by Northcott et al. (2017) and Sekhon et al (2015); participants were asked to select all options that applied to them. An "other" option was also included to allow participants to provide comment on other barriers.

Two questions asked participants to comment on whether a better understanding of mental health would affect how they provided hearing related services to their clients and whether they believed their clients would be open to engaging in conversations regarding mental health.

A preliminary version of the survey was reviewed by four mental health consultants and five clinical audiologists to ensure the appropriateness of the items and the ecological validity of the behaviour depicted in the scenarios. Minor adjustments were made to the survey following the review, including removal of items to reduce the length of the survey and rewording replacing the word "counselling" with the phrase "providing emotional support"

\section{Procedure}


Australian audiologists who had experience working with adults with hearing loss in any setting and at any stage of their career were invited to participate in the study. Participants were recruited from a series of audiology training workshops conducted by the peak professional audiology body of Australia (Audiology Australia) in Perth (Western Australia), Adelaide (South Australia) and Hobart (Tasmania) in November 2017. Printed copies of the participant information sheet and survey set were made available to all clinicians at the workshops, with completed surveys placed in a box to ensure anonymity of responses. Paper versions of the survey also provided a URL to an electronic version of the survey (using Qualtrics) for those clinicians who preferred to complete the survey online. An invitation to participate in the study was also advertised in the quarterly electronic magazine that is emailed to all members of Audiology Australia in the same month as the workshops described above. This invitation included a URL to the electronic version of the survey.

Participants who completed the printed version of the survey during the workshops were given approximately six hours to complete the surveys as they were collected at the end of the day. The online version of the survey was open for one month. No reminders were sent to potential participants. No attempts were made to monitor whether participants completed the surveys independently or with their colleagues (as may have been the case for those recruited during the workshops). All returned surveys were included in the analyses, including those partially completed.

\section{Data analysis}

Data from Qualtrics were exported to a Microsoft Excel file and responses in paper format manually entered into the Excel spread sheet. Quantitative analyses were performed 
using SPSS Statistics (version 21.0, Armonk, NY: IBM Corp). Qualitative content analyses were performed using Microsoft Excel.

Knowledge of depression. Participant responses to the knowledge of mental health section were explored using descriptive statistics (counts and percentages). Group differences in depression knowledge scores were examined using univariate linear regression. Variables investigated included participant age, gender, years of clinical experience, state / territory of employment, personal experience with mental illness, and whether the audiologist routinely ask their audiological clients about mental health concerns.

Beliefs and practices. Participants' clinical behaviours responding to presentations of emotional distress or mental health disorders during audiology appointments were investigated in two parts: 1) providing emotional support to people with hearing loss and 2) providing mental health support to people with hearing loss. Participants' responses to the multiple choice items within these sections were explored using descriptive statistics (counts and percentages). Chi-Square analyses were used to investigate whether participant age or years of experience influenced whether they routinely asked their audiology clients about their mental health status.

Audiologists' beliefs about delivering emotional support to people with hearing loss were also examined using two open ended questions: 1 ) on whether they believe that a better understanding of the emotional and mental health status of clients with hearing loss would affect the way in which they provide hearing related services and/or the outcomes received by clients, and 2) how they perceived the attitudes of people with hearing loss towards engaging in conversations regarding mental health and emotional well-being during audiology appointments. Content analysis was used to analyse the responses to these two 
open ended questions (Graneheim \& Lundman, 2004). This qualitative analysis involved: (1) reading and clarifying participants' answers to survey questions; (2) identifying meaning units within the data (identifying individual words/phrases within the data, yet still retaining their original meaning and context; (3) coding meaning units by grouping together those most closely related; and (4) grouping coded meaning units into categories. Peer debriefing was used to improve the rigour of the qualitative content analysis. One author completed the initial content analysis (EL). Two other members of the research team (CB \& RB) then crosschecked all of the analysed data to strengthen the accuracy of the coding. Categories and meaning units were tabulated, with the number of participants contributing to each meaning unit provided.

\section{RESULTS}

Participants included 95 Australian audiologists. It is not possible to provide a response rate given the snowballing methods used for recruitment. However, this represents approximately $3 \%$ of all audiologists in Australia. Participants included representatives from all states and territories in Australia, most were female reflecting the gender balance in the audiology profession in Australia (Table 1). Participants were asked to indicate what their areas of clinical specialty were, and given the choice to select multiple areas. From greatest to least, participants indicated their areas of clinical specialty were in adult audiological rehabilitation $(79 \%, n=75)$, tinnitus $(29 \%, n=28)$, implantable device $(18 \%, n=17)$, paediatric audiological rehabilitation $(13 \%, n=12)$, and balance and vestibular $(6 \%, n=6)$. Twenty $(21 \%)$ participants indicated other areas of clinical specialty ranging from paediatric diagnostics, newborn screening, auditory processing, outreach, hyperacusis management, aboriginal ear health, communication training, occupational hearing loss, and 
governance and policy. The majority of respondents $(83.5 \%, n=76)$ indicated they, or someone close to them, had experienced mental health issues. The majority of participants $(80 \%, n=78)$ indicated having received training in delivering basic counselling techniques, $68.4 \%(n=65)$ in social approaches to delivering emotional support (e.g. facilitating peer support; working with the family), and $52.6 \%(n=50)$ in the use of specific therapeutic methods for addressing mental health concerns (Figure 1).

\section{Knowledge of depression}

The majority of participants $(81 \%, n=77)$ completed the survey section evaluating knowledge of depression symptoms, causes and treatments. The mean Knowledge of Late Life Depression Scale - Revised (KLLD-R) score was 29.44 (on a scale of 10 to 40, SD =2.92), with a mean item score of 2.97. The mean knowledge of depression score (including the KLLDS-R and the six additional items relating specifically to adults with hearing loss) was 48.10 (on a scale from 16 to $64, S D=5.23$ ) (Table 2 ). No statistically significant relationship was found between total knowledge of depression scores and participant age $(p=0.809)$, gender $(p=0.844)$, years of clinical experience $(p=0.996)$, area of clinical speciality $(p=$ $0.993)$, or state or territory in which they usually work $(p=0.607)$. Knowledge of depression was not significantly different between those participants who had personal experience with mental illness ( $83.5 \%$ of respondents, $\mathrm{n}=76$ ) and those who had not $(p=0.571)$; nor between those participants who indicated that they routinely ask their audiology clients about their mental well-being $(31.6 \%, \mathrm{n}=30)$ and those that do not $(p=0.132)$.

\section{Beliefs and practices}


Thirty respondents (31.6\%) indicated they routinely asked audiology clients whether they were concerned about their mental well-being or whether they had recently experienced anxiety or depression. Whether participants asked their clients about their mental health status or not did not differ across age groups $\left[X^{2}(4)=3.540, p=0.472\right]$ or years of clinical experience $\left[X^{2}(4)=1.690, p=0.793\right]$. Reasons for not asking about mental well-being provided in the free text included "Only in relation to tinnitus", "May take offense if become too personal in an audiology set up", "I feel uncomfortable addressing more extreme responses such as suicidal thoughts", and "Personally I would resent being asked this unless it was relevant and connected to something under discussion".

The majority of participants $(96 \%, n=74)$ indicated that they would like to develop their knowledge and skills associated with mental health and emotional well-being. The comments provided by participants in this section included "it would be nice to feel more equipped to handle those clients", "I think it's an essential component of the audiologist's role", and "there should be more offered in this area through continuing education opportunities".

Providing emotional support. The most common barriers to delivering emotional support to people with hearing loss were feeling under-skilled / lack of training $(64.5 \%, \mathrm{n}=$ 49), worry that I may get 'out of my depth' $(56.6 \%, n=43)$ and time / caseload pressures $(55.3 \%, n=42)$ (Figure 2). Almost one third $(n=24)$ of participants indicated that they believed the provision of emotional support was not within an audiologist's scope of practice. Fifteen participants indicated Other, with comments including "lack of consistent referral options", "I don't know who to send them to", "I would only start the discussion if I felt the client would be open to discussion", and "people don't expect their audiologist to start asking about those things. I might put my clients off". 
The most common enablers to improving the delivery of emotional support to people with hearing loss were provision of more training $(83.1 \%, \mathrm{n}=64)$, knowing who and how to refer for mental health services $(77.9 \%, n=60)$, being able to access support / supervision $(62.3 \%, \mathrm{n}=48)$, and having adequate time to address emotional well-being $(51.9 \%, n=40)$ (Figure 3). Seven participants described Other enablers, including "feedback from mental health professionals about when appropriate referrals have been made, reports about diagnoses, treatment, etc. (i.e. being kept in the loop about client management)", and "having dealt with people with mental illness and the poor response of medical practitioners it's very hard to have confidence in referrals".

Providing mental health support. The majority of audiologists were extremely likely to refer a client to a mental health practitioner if they presented with suicidal thoughts and unlikely to refer a client reporting loneliness (Figure 4). The most common barriers to referring to mental health professionals included not knowing who to refer to $(54.7 \%, n=$ 41), not knowing when to make a referral $(49.3 \%, \mathrm{n}=37)$, or not knowing how to make a referral (38.6\%, $n=29$ ) (Figure 5). Twenty-four participants indicated Other, with many comments describing negative experiences with other health professionals when referring for mental health concerns. One participant wrote "in my experience there is limited access to mental health practitioner for elderly people". Another wrote "Finding mental health practitioners who have appropriate treatment protocols for hearing related issues are next to non-existent". Another wrote "the mental health practitioners in my town are [expletive] hopeless".

Participants were asked to comment on whether they believe that a better understanding of the emotional and mental health status of clients with hearing loss would affect the way in which they provide hearing related services and/or the outcomes received 
by clients. Seventy-five percent of respondents $(n=54)$ indicated it would be good to have a better understanding of clients' emotional and mental health status, $1 \%(n=4)$ indicated that there would be no benefit, $19.4 \%(n=14)$ provided comment that did not provide a definitive yes or no. The open-ended responses put forward by participants described improved understanding of client needs, personalisation of care, interdisciplinary services and outcomes that address the client as a whole person (Table 3).

Participants were asked how they perceived the attitudes of people with hearing loss towards engaging in conversations regarding emotional well-being and mental health during audiology appointments. Thirty-six percent of participants $(n=27)$ provided positive comments, $26 \%(n=19)$ provided negative comments, $31 \%(n=23)$ described it being client specific (variable), and $7 \%(n=5)$ said they were unsure. The open ended responses put forward by participants described the audiologists' perceptions as to the reasons behind clients' willingness or reluctance to engage in conversations relating to mental health; these comments emphasised the importance of the client-clinician relationship (Table 4).

\section{DISCUSSION}

We surveyed 95 Australian audiologists on their knowledge, beliefs and practices in addressing the emotional and mental health needs of adults with hearing loss. Findings indicate that Australian audiologists hold a moderate level of knowledge of late-life depression but require more training and support to facilitate provision of emotional and mental health support during audiology consultations. Specific organisational barriers identified by participants included insufficient time, funding and mentorship/support.

\section{Knowledge of depression}


Audiologists participating in the present study demonstrated a moderate knowledge of facts, myths and symptoms of depression, comparable to previous studies investigating nursing staffs' knowledge of depression in aged care settings. Where Davidson et al. (2009) reported mean Knowledge of Late-Life Depression (KLLD) total scores ranging from 28.82 to 32.38 for carers and registered nurses, the mean total score for audiologists involved in this study was 29.44. Similarly, where Karantzas et al. (2012) reported mean item scores of 2.84 for residential and 2.90 for community nurses and carers in aged care settings, the mean item score for audiologists involved in this study was 2.97. Davison et al. (2009) described this level of knowledge as moderate, suggesting that workplace experience is insufficient for knowledge development, and called for targeted training to improve the detection and management of late-life depression by allied health professionals. Prior mental health training was found to be associated with significantly higher levels of knowledge and confidence in managing psychological well-being in nursing staff working in residential and community aged care settings (Davison et al., 2009), and speech-and-language therapists (Northcott et al., 2017; Sekhon et al., 2015). The majority (96\%) of audiologists in the current study indicated that they would like to develop their knowledge and skills associated with mental health and emotional well-being, suggesting that targeted mental health training would be widely accepted by practicing audiologists.

\section{Beliefs and practices}

Providing emotional support. Almost one quarter of audiologists in the present study indicated that providing emotional support during clinical consultations was not within an audiologists' scope of practice. Similar views were reported by students and practicing audiologists in the USA, describing uncertainty regarding the role of the audiologist in 
discussing and addressing mental health related concerns with clients in the audiology setting (Muñoz, McLeod, Pitt, Preston, Shelton, \& Twohig, 2017). These beliefs clearly contradict clinical guidelines promoting the importance of considering the social, psychosocial and psychological needs of clients during the process of audiological rehabilitation (American Speech-Language-Hearing Association, 2018; Audiology Australia, 2013). Beck and Kulzer (2018) provide a detailed discussion of the audiologists' scope of practice and describe how and why emotional support (also described as emotion-focussed counselling) is an important aspect of quality audiological services. They further provide a comparison between the scope of practice for audiologists and for counsellors trained in the provision of psychotherapy-based counselling techniques, highlighting the overlaps and differences in practice. The emerging literature from both fields (audiology and counselling) indicate that it is an essential role of the clinician to provide counselling related to psychosocial adjustment to disability, and thus, clients are best served by clinicians who are well trained and specialized in the disability or condition, such as audiologists in the case of adults with hearing loss (Beck \& Kulzer, 2018). We emphasise that improving audiologists' knowledge and skills relating to the provision of mental health support does not negate, but rather enhance their ability to accurately identify and refer clients who would benefit from therapeutic interventions provided by trained mental health practitioners.

Although more than $80 \%$ of audiologists in the current study indicated that they had received some training in the delivery of emotional support, the two most common barriers to providing emotional support during audiology consultations were feeling under skilled and worry that I may get 'out of my depth', suggesting a need for further training and skill development. A recent international Delphi study identified 64 competency items (16 
knowledge-based, 35 skill-based, and 13 attitudinal items) deemed important for audiologists to possess to provide effective audiologic counselling (Meibos, 2019). Meibos (2019) further demonstrated that audiology training programs do not encompass adequate training on the majority of these items, suggesting that practicing clinical audiologists are under-skilled in many of these areas.

Providing mental health support. Three quarters of the audiologists participating in the current study indicated it would be good to have a better understanding of clients' emotional and mental health status. The potential benefits of this, as described by participants, included improved understanding of client needs, personalisation of care, offering interdisciplinary services, and broadening outcomes to address the client as a whole person. However, participants' perceptions regarding their clients' attitudes towards discussing mental health during audiology appointments varied, with one quarter providing positive comments, one quarter providing negative comments, and the rest describing it as being client specific, or being unsure. Audiologists emphasised the importance of the clientclinician relationship influencing their clients' willingness and/or reluctance to engage in conversations relating to mental health. The personal relationship that develops between the client and clinician is termed the therapeutic relationship, and in the case of audiological rehabilitation it is fostered by trust and loyalty (Grenness et al., 2014). Studies exploring the therapeutic relationship in the audiology setting have described its influence on decision making (Laplante-Lévesque et al., 2010), hearing aid uptake (Poost-Foroosh et al., 2011), the acquisition of hearing aid management skills and knowledge (Bennett et al., 2019), and help seeking for problems relating to hearing aid use (Bennett et al., 2018). Therapeutic 
relationships have the capacity to transform and enrich the client experience of audiological care (Clark \& English, 2013).

Although the majority of audiologists indicated that they would refer on a client presenting with mental health symptoms, the majority of audiologists also indicated that they did not know who to refer to, when to make a referral, or how to make a referral. Uncertainty regarding mental health referral pathways is not unique to audiology practice, in a recent study, $34 \%$ of UK speech pathologists identified unclear referral guidelines as being a significant barrier to the referral of adults with aphasia for mental health services (Northcott et al., 2017). The role of the audiologist in mental health symptom identification is clearly stated in the American Speech-Language-Hearing Association scope of practice, describing the administration and interpretation of diagnostic screening, including "measures of mental health" (American Speech-Language-Hearing Association, 2018). However, the role of the audiologist in referral for mental health is not clear, as specific guidance is not provided. Development of clinical guidelines describing how to evaluate, discuss, and address mental health (including referral pathways) during audiological consultations will assist audiologists in better understanding their role and responsibilities. Furthermore, opportunities for interdisciplinary collaboration between the mental health counselling and audiology professions should be encouraged, for them to learn from each other's areas of expertise to expand upon and improve our clinical services. Audiologists may consider championing this in their communities by making contact with local mental health practitioners, sharing their knowledge, requesting collaborative client care plans and developing mutually beneficial practice alliances. 
Organisational barriers. In addition to knowledge and training deficits, audiologists also raised organisational barriers to the provision of emotional and mental health support during audiological appointments. Organisational barriers have previously been reported by audiologists as key barriers to incorporating evidence-based client-centred approaches in audiology practices; specifically, a lack of resources, guidelines and regulations (Danermark, 2014). Mirroring the enablers identified in Northcott et al. (2017) and Sekhon et al. (2015), audiologists in the current study highlighted the need for increased appointment times to enable provision of emotional support, access to mental health services, supervision/support from their peers and managers, and funding for provision of these services. Where audiology service providers traditionally structure their fees on the hearing aid model selected to support the rehabilitation program, there has recently been a call for "unbundling" of fee structures, so as to charge for the hearing aid as a separate item to the consultation(s) (Windmill, Bishop, Elkins, Johnson, \& Sturdivant, 2016). This unbundling approach accounts for variation in the complexity of the client needs, the complexity of the services rendered, and the amount of time or number of appointments required to complete the rehabilitation program. Unbundling could thus be a means to allow for the additional time and funding required when providing emotional and mental health support and referral.

\section{Clinical Implications}

Adults with hearing loss experience an increased risk of developing emotional and mental health disorders (Weinstein, Sirow, \& Moser, 2016; Lawrence et al., 2019). Audiologists therefore have a clinical responsibility to ensure they are sufficiently trained to lead discussions with their clients about their emotional and mental health, and potentially 
refer clients to medical practitioners and mental health professionals. Counselling skills training workshops are shown to be effective for improving audiologists' communication skills (Muñoz, Ong, Borrie, Nelson, \& Twohig, 2017). It is possible that targeted mental health training may thus be effective in improving audiologists' skills in managing the mental health needs of their clients (Davison et al., 2009; Northcott et al. 2017; Sekhon et al., 2015); however, the effectiveness of such programs on the clinical behaviours of audiologists requires further investigation.

One strategy that has been used to successfully improve knowledge, create awareness and address stigma associated with mental health conditions is Mental Health First Aid (MHFA) (Hanisch et al., 2016; Jorm, Kitchener, Fischer, \& Cvetkovski, 2010). MHFA courses educate participants on the initial help to give people with developing mental health issues, including how to have those "difficult conversations", and the assistance to give people in mental health crises. These programs include a simple five-step plan of management for supporting people with mental health issues, consisting of (1) Assess risk of suicide or harm, (2) Listen non-judgementally, (3) Give reassurance and information, (4) Encourage person to get appropriate professional help and (5) Encourage self-help strategies (Kitchener \& Jorm, 2006). Two-day MHFA courses are freely available to students in most major Australian universities, and are also accessible through various universities and mental health institutions in Canada, Finland, USA, Scotland, Ireland, Singapore and Hong Kong (Kitchener \& Jorm, 2008). Audiology program coordinators could look to include MHFA as compulsory training for students. Peak professional bodies could look at promoting MHFA training for practicing audiologists. Future research could look towards tailoring existing MHFA programs to focus on the emotional and mental health issues more commonly experienced by clients in the audiology setting. 


\section{Limitations and future research}

This preliminary investigation into audiologists' knowledge, beliefs and practices in addressing the emotional and mental health needs of adults with hearing loss focussed on the Australian context. The knowledge, beliefs and practices of audiologists in other countries is unknown, and could be investigated in line with the findings of the present study. One limitation of research via self-selecting survey is the effects of response bias. We estimate that approximately $3 \%$ of all Australian audiologists participated in the study, with a mixed sample regards to age, gender, years of experience, location of employment, and area of clinical speciality. One possible limitation was the geographical spread of participants; $70 \%$ of respondents came from three of the seven states/territories (probably because of the recruitment methods used), and therefore the findings may not be reflective of the views of audiologists in less represented areas. Participants self-selected to participate, and it is likely that the clinicians more interested in mental health would be inclined to volunteer to participate in the study. Additionally, the effects of social desirability response bias should be taken into account; that is, the tendency for participants to present a favourable image of themselves (van de Mortel, 2008). Response biases of this nature may have been mitigated by the anonymity of the survey, giving respondents freedom to raise controversial viewpoints or acknowledge clinical weaknesses that they might avoid disclosing in front of peers. Along the same thought, participants self-reported their clinical behaviours relating to mental health management and so we do not know what is actually happening during clinical encounters. Nonetheless, this self-report survey methodology enabled us to canvas a broad range of views and provide direction for future research. 
The lack of validated surveys for evaluating allied health professionals knowledge of mental health, especially with respect to adults with hearing loss, posed a limitation in the current study. Thus audiologists' knowledge of depression was investigated using the KLLDR survey, with the inclusion of six additional questions focussing on mental health issues experienced by adults with hearing loss. The survey items and format were not validated prior to use in this study, as we had no intention on recommending their use beyond this scoping study. We only wanted to conduct an exploratory study providing an indication of audiologists' knowledge of depression, and thus scores and associations should be interpreted with caution. A further limitation was that we chose not to provide definitions of concepts such as emotional well-being or mental health in the survey. This allowed us to explore how audiologists themselves interpret these concepts in their free-text responses. However, it is possible that respondent's interpreted core constructs in different ways. Future research may consider exploring audiologists understanding of these terms both within and outside of the audiology context.

Treating the mental health condition is outside of the audiologists' scope of practise, and thus referral to specialised mental health practitioners is an important part of the role of the audiologist. Clinical interventions designed to improve referral processes for mental health conditions in older adults include screening instruments (Excellence \& Britain, 2004; Pignone et al., 2002), clinical decision making tools (Adli et al., 2017), and stepped or collaborative care approaches (Katon et al., 2005; Pomerantz et al., 2010; Unützer et al., 2008; van Straten, Hill, Richards, \& Cuijpers, 2015); however, the applications of these in the audiology setting is unknown and requires further investigation.

\section{CONCLUSION}


As allied health professionals, audiologists play a vital role in contributing to the holistic health of their clients and therefore have a clinical responsibility to ensure they are sufficiently trained to address the mental health needs of their clients. However, results from this study indicate that Australian audiologists have a moderate level of knowledge regarding depression in older adults. Knowledge, skills and time were identified as the key areas that require attention in order to allow audiologists to address the emotional and mental health needs of adults with hearing loss. The majority of participants in the current study agreed that hearing related services and the associated client outcomes would be enhanced through clinician knowledge and understanding of the emotional and mental health status of individual clients. Encouragingly, almost all participants indicated that they would like to develop their knowledge and skills associated with the provision of emotional and mental health support in the audiology setting, indicating that there is scope for expanding audiological services to include consideration and management of the psychological factors contributing to communication issues, and caused by hearing loss.

\section{Acknowledgements}

The authors would like to acknowledge the participants for devoting their time to this study. The authors report no declarations of interest. Portions of this article were presented at the Audiology Australia National Congress, Sydney NSW, May, 2018.

\section{References}

Adli, M., Wiethoff, K., Baghai, T. C., Fisher, R., Seemüller, F., Laakmann, G., . . Laux, G. (2017). How effective is algorithm-guided treatment for depressed inpatients? Results from the 
randomized controlled multicenter German Algorithm Project 3 trial. International Journal of Neuropsychopharmacology, 20(9), 721-730.

American Speech-Language-Hearing Association. (2018). Scope of Practice in Audiology. Retrieved from https://www.asha.org/policy/sp2018-00353/

Audiology Australia. (2013). Audiology Australia Professional Practice Standards - Part B Clinical Standards. Retrieved from http://www.audiology.asn.au/standardsdownloads/Clinical\%20Standards\%20-\%20whole\%20document\%20July13\%201.pdf

Australian Bureau of Statistics. (2008). National Survey of Mental Health and Wellbeing: Summary of Results, 2007. Cat. no. (4326.0). Canberra: ABS

Beck, K. \& Kulzer, J. (2018) Teaching counseling microskills to audiology students: recommendations from professional counseling educators. Seminars in Hearing, 39:1.

Bennett, R. J., Laplante-Lévesque, A., \& Eikelboom, R. H. (2018). How do hearing aid owners respond to hearing aid problems? Ear and Hearing, 40(1), 77-87.

Bennett, R. J., Meyer, C. J. \& Eikelboom, R. H. (2019). How do hearing aid owners acquire hearing aid management skills? Journal of the American Academy of Audiology, 30, 516-532.

Broese van Groenou, M., Hoogendijk, E. O., \& van Tilburg, T. G. (2013). Continued and new personal relationships in later life: Differential effects of health. Journal of Aging and Health, 25(2), 274-295.

Byers, A. L., Yaffe, K., Covinsky, K. E., Friedman, M. B., \& Bruce, M. L. (2010). High occurrence of mood and anxiety disorders among older adults: The National Comorbidity Survey Replication. Archives of general psychiatry, 67(5), 489-496.

Clark, J. G., \& English, K. M. (2013). Counseling-infused audiologic care. Pearson Higher Ed. Danermark, B. (2014). Patient-centred audiological rehabilitation: facilitating and hindering factors for implementation. ENT Audiol News, 23(23), 71-72. 
Davison, T. E., McCabe, M. P., Mellor, D., Karantzas, G., \& George, K. (2009). Knowledge of late-life depression: an empirical investigation of aged care staff. Aging \& Mental Health, 13(4), 577586.

Ekberg, K., Barr, C., \& Hickson, L. (2017). Difficult conversations: talking about cost in audiology consultations with older adults. Int J Audiol, 56(11), 854-861.

Ekberg, K., Grenness, C., \& Hickson, L. (2014). Addressing patients' psychosocial concerns regarding hearing aids within audiology appointments for older adults. American Journal of Audiology, 23(3), 337-350.

English, K., Naeve-Velguth, S., Rall, E., Uyehara-Isono, J., \& Pittman, A. (2007). Development of an instrument to evaluate audiologic counseling skills. Journal of the American Academy of Audiology, 18(8), 675-687.

English, K., Rojeski, T., \& Branham, K. (2000). Acquiring counseling skills in mid-career: Outcomes of a distance education course for practicing audiologists. Journal of the American Academy of Audiology, 11(2), 84-90.

Graneheim, U. H., \& Lundman, B. (2004). Qualitative content analysis in nursing research: concepts, procedures and measures to achieve trustworthiness. Nurse Education Today, 24(2), 105112.

Grenness, C., Hickson, L., Laplante-Lévesque, A., \& Davidson, B. (2014). Patient-centred care: A review for rehabilitative audiologists. International Journal of Audiology, 53(S1), S60-S67.

Grenness, C., Hickson, L., Laplante-Levesque, A., Meyer, C., \& Davidson, B. (2015). The nature of communication throughout diagnosis and management planning in initial audiologic rehabilitation consultations. Journal of the American Academy of Audiology, 26(1), 36-50.

Hanisch, S. E., Twomey, C. D., Szeto, A. C., Birner, U. W., Nowak, D., \& Sabariego, C. (2016). The effectiveness of interventions targeting the stigma of mental illness at the workplace: a systematic review. BMC Psychiatry, 16(1), 1. 
Jorm, A. F., Kitchener, B. A., Fischer, J.-A., \& Cvetkovski, S. (2010). Mental health first aid training by e-learning: a randomized controlled trial. Australian and New Zealand Journal of Psychiatry, 44(12), 1072-1081.

Karantzas, G. C., Davison, T. E., McCabe, M. P., Mellor, D., \& Beaton, P. (2012). Measuring carers' knowledge of depression in aged care settings: The Knowledge of Late Life Depression Scale-Revised. Journal of Affective Disorders, 138(3), 417-424.

Katon, W. J., Schoenbaum, M., Fan, M.-Y., Callahan, C. M., Williams, J., Hunkeler, E., . . Unützer, J. (2005). Cost-effectiveness of improving primary care treatment of late-life depression. Archives of General Psychiatry, 62(12), 1313-1320.

Kitchener, B. A., \& Jorm, A. F. (2006). Mental health first aid training: review of evaluation studies. Australian \& New Zealand Journal of Psychiatry, 40(1), 6-8.

Kitchener, B. A., \& Jorm, A. F. (2008). Mental Health First Aid: an international programme for early intervention. Early Intervention in Psychiatry, 2(1), 55-61.

Kramer, S. E., Kapteyn, T. S., Kuik, D. J., \& Deeg, D. J. (2002). The association of hearing impairment and chronic diseases with psychosocial health status in older age. Journal of Aging and Health, 14(1), 122-137.

Laplante-Lévesque, A., Hickson, L. \& Worrall, L. (2010). A qualitative study of shared decision making in rehabilitative audiology. Journal of the American Academy of Rehabilitative Audiology, 43, 27-43.

Lawrence, B. J., Jayakody, D. M., Eikelboom, R. H., Bennett, R. J., Gasson, N., \& Friedland, P. L. (2019). Age-related hearing loss and depression in older adults: A systematic review and metaanalysis. The Gerontologist, 2019 Mar 5. pii: gnz009. [Epub ahead of print].

Meibos, A., Munoz, K., Schultz, J., Price, T., Whicker, J. J., Caballero, A., \& Graham, L. (2017). Counselling users of hearing technology: A comprehensive literature review. International Journal of Audiology, 56(12), 903-908. 
Meibos, A., Muñoz, K., \& Twohig, M. (2019). Counseling Competencies in Audiology: A Modified Delphi Study. American Journal of Audiology, 28(2), 285-299.

Mick, P., Kawachi, I., \& Lin, F. R. (2014). The association between hearing loss and social isolation in older adults. Otolaryngology--Head and Neck Surgery, 150(3), 378-384.

Mikkola, T. M., Polku, H., Portegijs, E., Rantakokko, M., Tsai, L. T., Rantanen, T., \& Viljanen, A. (2016). Self-reported hearing is associated with time spent out-of-home and withdrawal from leisure activities in older community-dwelling adults. Aging Clinical and Experimental Research, 28(2), 297-302.

Muñoz, K., Ong, C. W., Borrie, S. A., Nelson, L. H., \& Twohig, M. P. (2017). Audiologists' communication behaviour during hearing device management appointments. International Journal of Audiology, 56(5), 328-336.

Muñoz, K., McLeod, H., Pitt, C., Preston, E., Shelton, T., \& Twohig, M. P. (2017). Recognizing emotional challenges of hearing loss. The Hearing Journal, 70(1), 34-37.

Northcott, S., Simpson, A., Moss, B., Ahmed, N., \& Hilari, K. (2017). How do speech-and-language therapists address the psychosocial well-being of people with aphasia? Results of a UK online survey. International Journal of Language \& Communication Disorders, 52(3), 356373.

Pignone, M. P., Gaynes, B. N., Rushton, J. L., Burchell, C. M., Orleans, C. T., Mulrow, C. D., \& Lohr, K. N. (2002). Screening for depression in adults: a summary of the evidence for the US Preventive Services Task Force. Annals of Internal Medicine, 136(10), 765-776.

Pomerantz, A. S., Shiner, B., Watts, B. V., Detzer, M. J., Kutter, C., Street, B., \& Scott, D. (2010). The White River model of colocated collaborative care: A platform for mental and behavioral health care in the medical home. Families, Systems, \& Health, 28(2), 114. 
Poost-Foroosh, L., Jennings, M. B., Shaw, L., Meston, C. N., \& Cheesman, M. F. (2011). Factors in client-clinician interaction that influence hearing aid adoption. Trends in Amplification, 15(3), 127-139.

Pronk, M., Deeg, D. J., \& Kramer, S. E. (2013). Hearing status in older persons: A significant determinant of depression and loneliness? Results from the Longitudinal Aging Study Amsterdam. American Journal of Audiology, 22(2), 316-320.

Reynolds, K., Pietrzak, R. H., El-Gabalawy, R., Mackenzie, C. S., \& Sareen, J. (2015). Prevalence of psychiatric disorders in US older adults: findings from a nationally representative survey. World Psychiatry, 14(1), 74-81.

Rutherford, B. R., Brewster, K., Golub, J. S., Kim, A. H., \& Roose, S. P. (2017). Sensation and psychiatry: linking age-related hearing loss to late-life depression and cognitive decline. American Journal of Psychiatry, 175(3), 215-224.

Sekhon, J. K., Douglas, J., \& Rose, M. L. (2015). Current Australian speech-language pathology practice in addressing psychological well-being in people with aphasia after stroke. International Journal of Speech-Language Pathology, 17(3), 252-262.

SPSS Inc. (2014). IBM @ SPSS Statistics (Version V21). Chicago, IL.

Unützer, J., Katon, W. J., Fan, M.-Y., Schoenbaum, M. C., Lin, E. H., Della Penna, R. D., \& Powers, D. (2008). Long-term cost effects of collaborative care for late-life depression. The American Journal of Managed Care, 14(2), 95.

van de Mortel, T. F. (2008). Faking it: social desirability response bias in self-report research. Australian Journal of Advanced Nursing, The, 25(4), 40.

van Straten, A., Hill, J., Richards, D., \& Cuijpers, P. (2015). Stepped care treatment delivery for depression: a systematic review and meta-analysis. Psychological Medicine, 45(2), 231-246.

Weinstein, B. E., Sirow, L. W., \& Moser, S. (2016). Relating hearing aid use to social and emotional loneliness in older adults. American Journal of Audiology, 25(1), 54-61. 
Windmill, I. M., Bishop, C., Elkins, A., Johnson, M. F., \& Sturdivant, G. (2016). Patient complexity charge matrix for audiology services: A new perspective on unbundling. Seminars in Hearing. 2016 May;37(2):148-60. 
Table 1. Cohort description $(n=95)$

\begin{tabular}{lc}
\hline Cohort description & $\begin{array}{c}\text { Number of } \\
\text { participants (\%) }\end{array}$ \\
\hline Age (years) & $19(20)$ \\
$20-29$ & $30(32)$ \\
$30-39$ & $26(27)$ \\
$40-49$ & $14(15)$ \\
$50-59$ & $6(6)$ \\
$60+$ & \\
Gender (n = 94) & \\
Female & $78(83)$ \\
Male & $16(17)$ \\
Years of experience as an audiologist & \\
less than 1 & \\
1-5 & $2(2)$ \\
6-10 & $26(27)$ \\
11-20 & $17(18)$ \\
more than 20 & $24(25)$ \\
Location of employment & $26(27)$ \\
Western Australia & \\
South Australia & \\
Tasmania & $38(40)$ \\
Queensland & $8(8)$ \\
New South Whales & $7(7)$ \\
Victoria & $11(12)$ \\
Northern Territory & $9(9)$ \\
Australian Capital Territory & $17(18)$ \\
\hline
\end{tabular}


Table 2. Mean scores for knowledge of depression questions; total scores and scores for each item. A higher score denotes a higher knowledge (item response scale 1-4).

\begin{tabular}{lc}
\hline Items describing knowledge of depression & Mean (SD) \\
\hline Sleep problems can be a symptom of depression* & 3.52 (0.50) \\
Depression is a normal reaction to the death of an older person's partner* & 2.25 (0.80) \\
Depression is common among aged care residents with dementia* & $1.83(0.66)$ \\
Tiredness can be a symptom of depression* & $3.31(0.54)$ \\
Depression is a normal reaction to the changes of old age* & $3.00(0.65)$ \\
It is common for depression to go undetected among older people* & $3.44(0.50)$ \\
Loss of interest in things previously enjoyed can be a sign of depression* & $3.44(0.55)$ \\
Most older people who have to sell their home and move into residential care will become depressed* & $2.86(0.70)$ \\
Older people with depression often report physical aches and pains rather than sadness* & $2.88(0.70)$ \\
Late life depression is associated with poorer recovery from physical illnesses* & $3.12(0.67)$ \\
There is a greater rate of depression in people with hearing loss than people with normal hearing & $3.16(0.74)$ \\
There is effective treatment available for late-life depression & $2.86(0.75)$ \\
Social isolation can contribute to depression & $3.65(0.62)$ \\
Older people don't commit suicide, it's more of a problem in younger age groups & $3.21(0.70)$ \\
It is normal for older people to talk about having nothing to live for & $2.86(0.79)$ \\
Hearing aids are sufficient to treat the symptoms of depression caused by hearing loss & $3.22(0.70)$ \\
\hline Total & $\mathbf{4 8 . 1 0 ( 5 . 2 3 )}$ \\
\hline * Item is from the Knowledge of Late Life Depression Scale - Revised (Karantzas et al., 2012)
\end{tabular}

* Item is from the Knowledge of Late Life Depression Scale - Revised (Karantzas et al., 2012) 
Table 3. Participants' perceptions on how their better understanding of the emotional and mental health status of clients with hearing loss would improve the way in which they provide hearing related services and/or the outcomes received by clients.

\section{Theme (number of participants responding with \\ Condensed meaning units (number of participants responding with this meaning unit)} this meaning unit)

Promote use of client counselling techniques (6)

Improved counselling skills (2)

Changes to the way in which results and recommendations are delivered (1)

Better at listening to client concerns (1)

A better understanding of how to interact with the client (1)

Improve understanding of the client (8)

Confidence to engage in conversations regarding mental health (1)

Affect how a client is viewed (1)

Improved understanding of client motivations and health-seeking behaviours (4)

Will help understand a client state of mind (2)

Personalisation of client care (16)

Assist with identifying personal communication goals (3)

Assist with setting realistic expectations (1)

Identify the need for additional support (1)

Improve delivery of communication training (1)

Identify the need for increased appointment times (2)

Arrange to have mental health concerns addressed before/during audiological rehabilitation (1)

Reduce emphasis on sales (1)

Determine rehabilitation priorities (1)

Adjust sequence of rehabilitation process (1)

Facilitate personalisation of the rehabilitation program (1)

Facilitate personalisation of follow up measures (1)

Better able to meet client needs (2)

Improve client outcomes (7)

Measure outcomes relevant to the client (2)

Improve client satisfaction (1)

Improve client well-being (1)

Better able to support clients (3)

Promote interdisciplinary care (7)

Refer to appropriate professionals (5)

Increase interdisciplinary interaction (2)

Encourage a holistic approach to client care (8)

Encourage holistic approach (7)

Broaden focus beyond hearing devices (1) 
Table 4. Participants' perceptions of the attitudes of people with hearing loss towards engaging in conversations regarding mental health and emotional well-being during audiology appointments.

Theme (number of participants responding with this meaning unit)

Clients are open and receptive to these discussions (58)

Clients are willing to discuss when related to hearing loss or relevant life circumstances (6)

Clients are willing to receive information (2)

Willingness depends on client personal and life factors (11)

Willingness depends on client-clinician relationship (18)

Willingness depends on whether the mental health concern is formally diagnosed (2)

\section{Condensed meaning units (number of participants responding with this meaning unit)}

Willing to discuss with audiologist (25)

Positive engagement (4)

They drop hints inviting the audiologist to probe further (1)

Okay with discussing mental health (2)

Happy to discuss mental health (2)

Comfortable talking about mental health (1)

Open to discussion regarding mental health (2)

Receptive to discussion (7)

Discussing mental health is a priority for some clients (2)

Older people are more willing (2)

Younger people are more open (1)

Responsive to sensitive and non-judgmental enquiries (4)

Positive response to audiologist initiation of discussion (5)

Non-specific / low-key discussion is accepted (2)

Discussion around impactful life circumstances (2)

Positive if framed in terms of hearing loss (2)

Willing to receive information (2)

Depends upon cultural background, gender etc. (2)

Depends on client life experiences (1)

Depends on client personality (8)

Happier to discuss with audiologist who has personal experience with these issues (1)

Dependent upon client / audiologist trust and relationship (9)

Depends on clinician ability to engage (5)

Responsive if confident that clinicians care (2)

Want to connect with audiologist (1)

Willing to discuss if formally diagnosed with a mental health condition (1)

Not willing to discuss if not formally diagnosed with a mental health condition (1) 
Clients appreciate the opportunity to discuss their concerns (14)

Audiologist perceived as a professional equip to address psychological needs (6)

Clients are unwilling to discuss mental health during audiology appointments (20)

Clients have a negative emotional response to this sort of discussion (18)

Discussions are not relevant or appropriate in this setting (10)
Happy to have someone listen to them (3)

Have little other opportunity to talk to people (5)

Keen to discuss problems and vent (6)

Audiologist perceived as a professional equipped to address psychological needs (2)

Audiologist viewed as a medical professional (1)

Prefer to talk to audiologist rather than mental health professional (3)

Information not volunteered (8)

Unwilling to talk about mental health in detail (2)

Not willing to discuss with audiologist (6)

Older people more resistant (3)

Men are less willing to talk about emotions (1)

Negative response (1)

Defensive response (2)

Avoidance / reluctance to discuss (8)

Client uncomfortable discussing (4)

Discussion perceived as too intrusive (2)

Clients get nervous talking about mental health (1)

Clients appear surprised when asked about mental health (1)

Considered outside of audiologist scope of practice (2)

Discussion on mental health is not considered appropriate in audiology appointments (2)

Audiologists are not perceived as a source of information or support for mental health care (1)

Mental health is considered to be irrelevant to audiology services (4) 
Figure 1. Amount of training received by audiologists in delivering emotional support

\section{Specific therapeutic...}

Social approaches (e.g....

Basic counselling...

None

CPD courses (1 day or less)

Short courses ( $>1$ day to less than 3 months)

Graduate or post-graduate certificate, diploma or equivalent

Figure 2. Barriers for delivering emotional support to people with hearing loss

\section{I feel under-skilled/ lack of training I worry that I may get 'out of my depth' \\ Time/ caseload pressures}

Lack of on-going specialist supervision in psychosocial..

Not within an audiologist's scope of practice Lack of funding for this service

Lack of research evidence on effective approaches for... Not the client's priority

Emotional support/counselling is a low priority where... I don't consider it part of my role
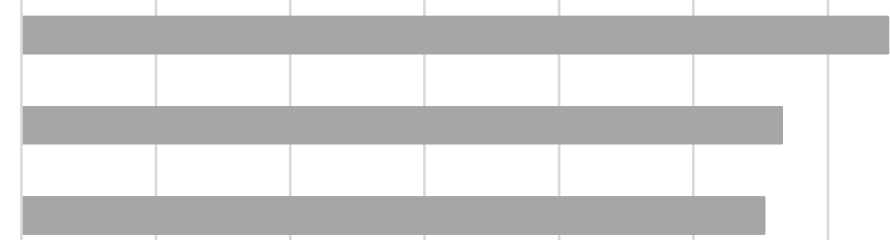

更

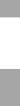

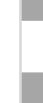

$$
\text { 西 }
$$


Figure 3. Enablers to improving the delivery of emotional support to people with hearing loss.

\section{Provision of more training}

Knowing who and how to refer for mental health services

Being able to access on-going supervision/ support from...

Having adequate time to address emotional well-being

Having a clinical decision tree tool/clinical management...

Access to funding for these services

My role definition encouraging me to address emotional...

Recognition from senior staff of the value of working on...

Not a priority for me

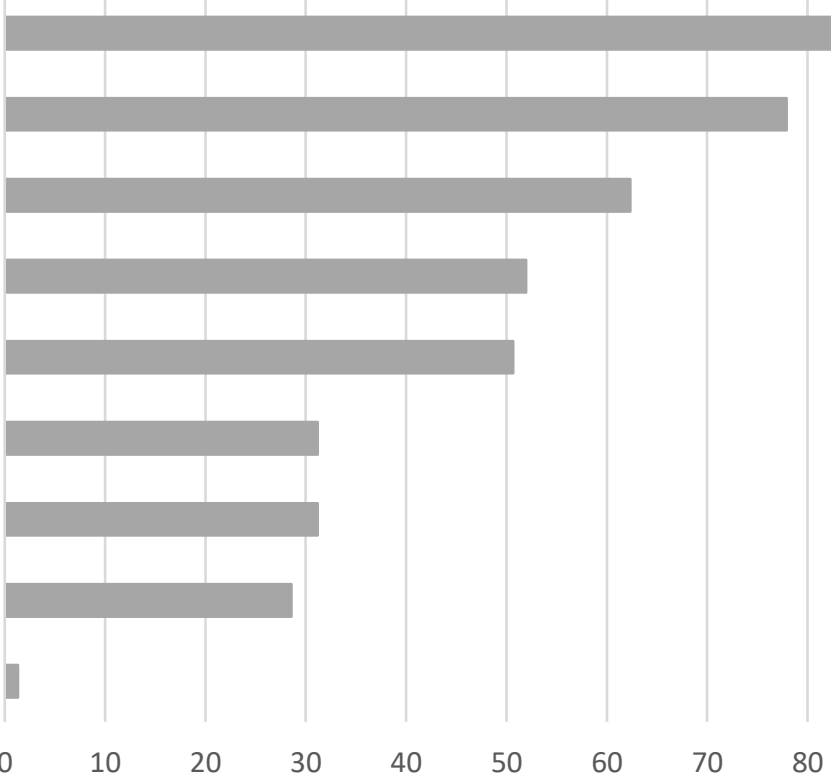

Percentage of respondents who selected this res

Figure 4. Likelihood of referring a client to a GP or mental health practitioner 


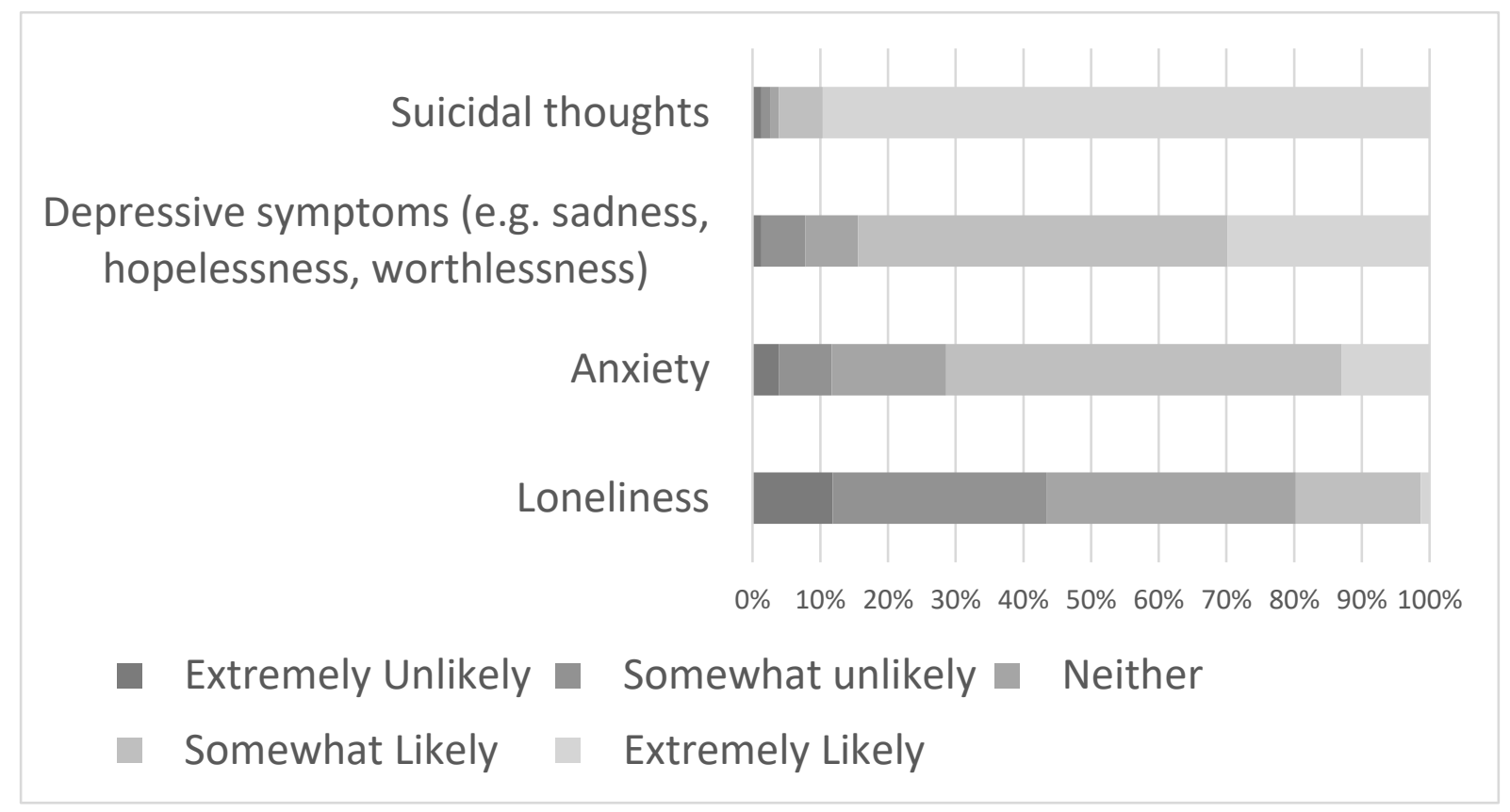

Figure 5. Barriers to making a refer to a mental health professional

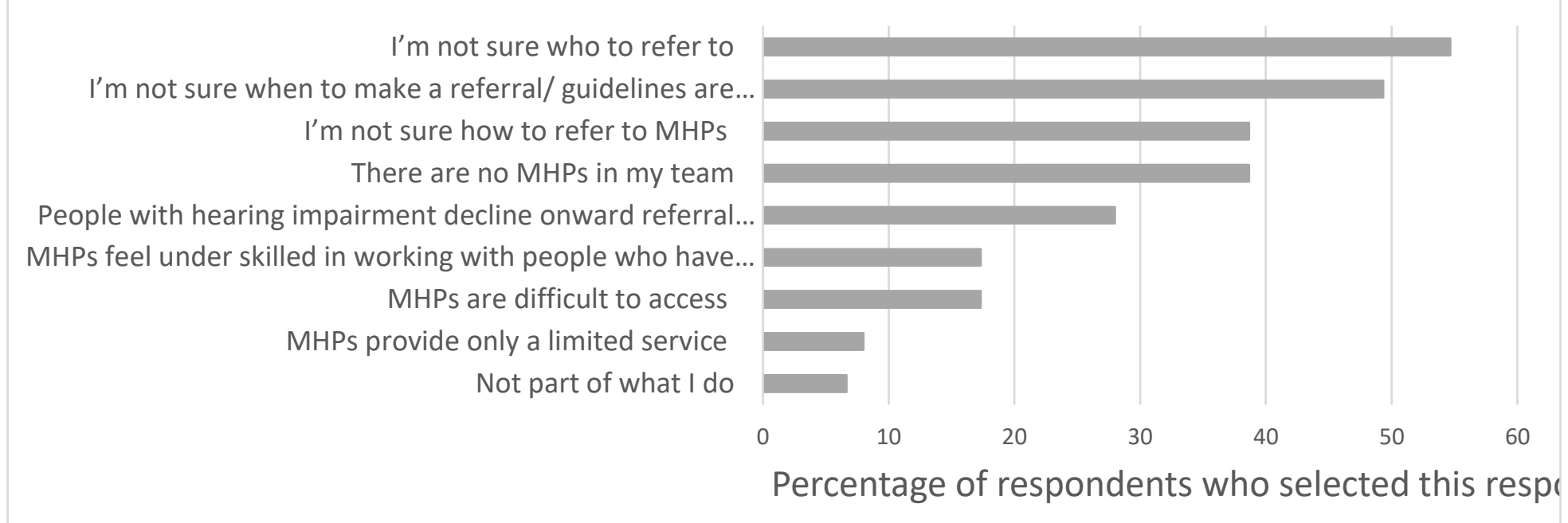

